\title{
Dependence of Cytoplasmic temperatures on the Vibrational energies of molecules in a eukaryotic cell Nucleus
}

Haider, M., ${ }^{\Psi}$, Akhtar, N., ${ }^{\phi}$ and Ali, M., ${ }^{\phi}$

$\Psi$ Rashid Latif Medical College, Lahore, Pakistan

$\phi$ Awan Clinic, Faisalabad, Pakistan

$\$$ Warsaw University of Technology, Poland

\begin{abstract}
This paper presents a statistical model to show the dependence of cytoplasmic temperatures on the vibrational kinetic energies of molecules in a eukaryotic cell nucleus. The probability distribution $P\left(E_{N}\right)$ of energy states of cell nucleus $E_{N}$ is derived using canonical ensemble framework and the vibrational energies of molecules are quadratic function of Temperature. It has been postulated that vibrational energies changes the reaction potentials of processes making certain reactions favorable. These favorable reactions explains the evolutionary processes such as mutation at molecular level. Natural Selection is simply just favorable reactions of molecules affected by the surrounding Temperature. The effect of temperature on vibrational energies of molecules can be effectively used to study cancerous mutations and astrobiology.
\end{abstract}




\section{Introduction}

Evolutionary processes such as natural selection and genetic drifts are well researched processes ${ }^{1}$ at every level of biological organizations from species to molecules ${ }^{2,3}$. However, the correspondence from molecular level to species level is not well established. The effects of environment on the processes at molecular levels gives the blue print of the effect that are observed at species level. To serve this purpose, this paper develops a simple statistical model to study the effect of environment (Temperature) at molecular level. In section 2, fluctuations in energy of eukaryotic cell by the temperature are modelled using canonical ensemble framework, which is effectively used in Physics specifically thermodynamics. The ingredient o eukaryotic cells are simple molecules arranged in specific arrangements. The fluctuations in energy states of cells causes the fluctuations in energy states of molecules. This paper specifically studies the dependence of temperature on the vibrational energies of the molecules, assuming chemical potential energy, translational and rotational kinetic energies constant. In section 3, it has been hypothesized using three postulates that fluctuations in vibrational energy of molecules controls the reaction potentials of processes at molecular level, making certain processes favorable. These favorable reactions have direct implication on the

processes such as natural selection at organism and specie level. In section 4, certain aspects of evolution theory at molecular level are discussed. Furthermore, it has been reasoned that mutation including cancerous mutation has dependence on the favorable reactions at molecular level caused by the cytoplasmic temperature. This model can be effectively used in astrobiology, where temperatures are significantly different from that on earth, making reaction potential at molecular level different. 


\section{Statistical Model of Eukaryotic Cell using Canonical Ensemble Framework}

Consider an arbitrary system with a fixed volume $\mathrm{V}$, energy $E_{\text {tot }}$ and microstates $\Omega_{\text {tot }} E_{\text {tot }}$. The system comprises of two subsystems 1 and 2. Subsystem 1 is the nucleus of the eukaryotic cell with energy $E_{N}$ and microstates $\Omega_{N} E_{N}$. Subsystem is the surrounding cytoplasm, containing other organelles with energy $E_{C}$ and microstates $\Omega_{C} E_{C}$. The total energy of the system is the sum of the energies of two subsystems i.e. $E_{t o t}=E_{N}+E_{C}$. The total microstates of the system are the product of the microstates of subsystems 1 and 2 i.e. $\Omega_{\text {tot }} E_{\text {tot }}=\Omega_{N} E_{N} \cdot \Omega_{C} E_{C}$.

\subsection{Assumptions}

Two subsystems 1 and 2 are in the state of thermodynamic equilibrium, where probability of all accessible microstates are equal. Furthermore, the energy of subsystem 1 is exceedingly less than the energy of the system i.e. $E_{t o t} \gg>E_{N}$. This assumptions corresponds to the fact that the fluctuations in the energy state of $E_{N}$ are governed by the properties of the subsystem 2. Energy states of subsystem 2 are continuum and $\frac{\partial \ln \Omega_{C} E_{t o t}}{\partial E_{t o t}}$ is well defined (Appendix A) and is equal to thermodynamic Beta $\beta$.

\subsection{Probability Distribution of Energy State fluctuations of $E_{N}$}

Based on the assumptions, the probability distribution $P\left(E_{N}\right)$ of energy state fluctuations of $E_{N}$ using canonical ensemble framework is given by the exponential distribution function of equation 1, where $\mathrm{Z}$ is the partition function given by $Z=\int e^{-E_{N} \beta} \rho\left(E_{N}\right) \partial\left(E_{N}\right)$ and $s$ is the ratio of microstates of subsystem 2 with energy and total number of microstates i.e. $s=\frac{\Omega_{C} E_{t o t}}{\Omega_{t o t} E_{t o t}}$. The derivation of equation 1 is given in Appendix A.

$P\left(E_{N}\right)=\frac{s \cdot e^{-E_{N} \beta}}{Z}$ 
Thermodynamic beta $\beta$ is given by $\frac{1}{k_{B} T}$, where $k_{B}$ is the Boltzmann constant and $T$ is the temperature of subsystem 2 given in Kelvins.

\subsection{Vibrational Energy Fluctuations of Molecules}

The energy of subsystem 1 which is the nucleus of eukaryotic is the sum of the energy of all the molecules of the nucleus given by equation 2, where $i$ is the number of molecules, $j$ is the type of molecule and $n_{j}$ is the total number of molecules of one particular type. The energy $e_{i_{j}}$ of the individual molecule is given by equation 3 , where $e_{i_{j}, p}$ is the chemical potential energy of the molecule, $e_{i_{j}, k_{T}}, e_{i_{j}, k_{R}}, e_{i_{j}, k_{V}}$ are the translational, rotational and vibrational kinetic energies of the molecule respectively.

$$
\begin{aligned}
& E_{N}=\sum_{i_{j}=1}^{n_{j}} e_{i_{j}} \\
& e_{i_{j}}=e_{i_{j}, p}+e_{i_{j}, k_{T}}+e_{i_{j}, k_{R}}+e_{i_{j}, k_{V}}
\end{aligned}
$$

Assuming the fluctuations in the energy states of $E_{N}$ contributes to the fluctuations in the vibrational kinetic energy of molecules $\sum_{i_{j}=1}^{n_{j}} e_{i_{j}, k_{V}}$, more than the other energies such that they can be assumed to be constant given by equation 4 .

$K=\sum_{i_{j}=1}^{n_{j}} e_{i_{j}, p}+\sum_{i_{j}=1}^{n_{j}} e_{i_{j}, k_{T}}+\sum_{i_{j}=1}^{n_{j}} e_{i_{j}, k_{R}}$

The probability distribution of energy states of $E_{N}$ in terms of vibrational kinetic energy of molecules can be written as equation 5, where $\mathrm{L}$ is the constant, using equation 4 is given by equation 6 $P\left(E_{N}\right)=\frac{L}{Z} e^{-\beta \sum_{i} \sum_{j} e_{i} e_{i_{j}, k_{V}}}$ 


\subsection{Average Vibrational Energy of Type 'a' molecule}

The thermodynamic free energy $\left\langle E_{N}\right\rangle$ of subsystem 1 is the expected value of the exponential probability distribution of equation 1 given by equation 7 . The average vibrational energy of the molecule $\overline{e_{k_{V}}}$ in a nucleus is given by equation 8 , where $\sum_{i_{j}=1}^{n_{j}} \overline{e_{l_{j}, k_{V}}}$ is the sum of all the average energies of the molecules of all type. Using equation 2, 4, 7 and 8, the average vibrational energy

$\overline{e_{a, k_{V}}}$ of an arbitrary type 'a' molecule can be calculated using equation 9 , where $\overline{e_{a, k_{V}}}=\frac{\sum_{i_{a}=1}^{n_{a}} \overline{e_{i_{a}, k_{V}}}}{n_{a}}$ and $\sum_{i_{j \neq k}=1}^{n_{j \neq k}} \overline{e_{i_{j}, k_{V}}}$ is the sum of vibrational energy of all molecules except Type 'a' molecules.

$\left\langle E_{N}\right\rangle=-\frac{\partial \ln Z}{\partial \beta}=k_{B} T^{2} \frac{\partial \ln Z}{\partial T}$

$\overline{e_{k_{V}}}=\frac{\sum_{i_{j}=1}^{n_{j}} \overline{e_{l_{j}, k_{V}}}}{\sum n_{j}}$

$\overline{e_{a, k_{V}}}=\frac{k_{B} T^{2} \frac{\partial \ln Z}{\partial T}-K-\left(\sum_{i_{j \neq k}=1}^{n_{j \neq k}} \overline{e_{i_{j}, k_{V}}}\right)}{n_{a}}$

The average vibrational energy of type ' $a$ ' molecules is clearly the quadratic function of Temperature T. As the value of temperature of subsystem 2 increases, the average vibrational energy of molecules in the cell nucleus increases. The Distribution of type 'a' molecules is given by the Gaussian distribution of equation 10 , where $(\sigma)^{2}$ is the variance of the distribution.

$P\left(e_{i_{a}, k_{\mathrm{V}}}\right)=\frac{1}{2 \pi(\sigma)^{2}} e^{-\frac{\left(e_{i_{a}, k_{\mathrm{V}}}-\overline{e_{\mathrm{a}, k_{\mathrm{V}}}}\right)^{2}}{2(\sigma)^{2}}}$ 
Type ' $a$ ' molecules have three spatial velocity directions, so the average vibrational energy of molecule is the combination of components in all three spatial direction $(\mathrm{z}, \mathrm{y}, \mathrm{x})$ given by equation 11

$\overline{e_{a, k_{V}}}=\sqrt{\left(\overline{e_{a, k_{Z}}}\right)^{2}+\left(\overline{e_{a, k_{V_{Y}}}}\right)^{2}+\left(\overline{e_{a, k_{X}}}\right)^{2}}$

The Gaussian distribution of molecules of in each spatial directions are given by equation 12, 13, 14, where $\left(\sigma_{\mathrm{z}}\right)^{2},\left(\sigma_{Y}\right)^{2},\left(\sigma_{X}\right)^{2}$ are the variances in each spatial direction.

$P\left(e_{i_{a}, k_{Z}}\right)=\frac{1}{2 \pi\left(\sigma_{\mathbf{Z}}\right)^{2}} e^{-\frac{\left(e_{i_{a}, k_{V}}-\overline{e_{\mathrm{a}, k_{V_{Z}}}}\right)^{2}}{2\left(\sigma_{\mathbf{Z}}\right)^{2}}}$

$P\left(e_{i_{a}, k_{V_{Y}}}\right)=\frac{1}{2 \pi\left(\sigma_{Y}\right)^{2}} e^{-\frac{\left(e_{i_{a}, k_{V_{Y}}}-\overline{e_{a, k_{V_{Y}}}}\right)^{2}}{2\left(\sigma_{Y}\right)^{2}}}$

$P\left(e_{i_{a}, k_{V_{X}}}\right)=\frac{1}{2 \pi\left(\sigma_{X}\right)^{2}} e^{-\frac{\left(e_{i_{a}, k_{V_{X}}}-\overline{e_{\mathrm{a}, k_{V}}}\right)^{2}}{2\left(\sigma_{X}\right)^{2}}}$

An arbitrary type 'a' molecule has three components of vibrational energy in each spatial direction. The variance of each component from the average energy is in turn the function of temperature. However, the degree of freedoms of each molecule of type 'a' is practically and computationally not feasible. To serve this purpose, this article only deals with the average vibrational energy $\overline{e_{a, k_{V}}}$ of the molecule specie. The average vibration energy corresponds to the oscillation frequency of the molecule. Assuming each molecule corresponds to a spring oscillating harmonically and obeys hooks law, the oscillation frequency $f$ of each molecule can be written as $f=\frac{1}{2 \pi} \sqrt{\frac{k}{\mu}}$, where $\mathrm{k}$ is the spring constant corresponding to the molecule type and $\mu$ is the rest mass. The displacement corresponding to this oscillation frequency is given by equation 15 , where $A$ is the amplitude and $\varphi$ is the initial phase angle. 
$u(t)=A \cos (2 \pi \mathrm{ft}+\varphi)$

The displacement $u(t)$ corresponds to the average vibrational energy $\overline{e_{a, k_{V}}}$, consequently, the displacements of each spatial component can be written in a similar way. The displacement $u(t)$ is the function of temperature of subsystem 2 or the surrounding of the nucleus.

\section{Favorable Reactions at Molecular Level}

This section qualitatively examines and hypothesize the significance of vibrational energies of molecules on the reaction processes. Consider a chemical reaction given by equation 16 , where $a, b$ are the reactants and $c$ is the product after the reaction. The average vibrational energies of reactants $a$ and $b$ are given $\overline{e_{a, k_{V}}}$ and $\overline{e_{b, k_{V}}}$ by respectively.

$a+b \rightarrow c$

The chemical reaction of equation 16 follows the following three postulates.

1. The reaction will only occur if the vibrational energies or oscillation frequencies of reactants are favorable for the forward reaction.

2. The kinetics of the reaction depends on the vibrational energies of the reactants.

3. The vibrational energy of the product depends on the vibrational energies of the reactants as well as the surrounding temperature $\mathrm{T}$.

Based on postulate 1, the favorable range for vibrational energy of reactants can be defined given by equation 17, where $j$ is the type of reactant, $\left[\overline{e_{a, k_{v}}}\right]_{\text {lower }}$ and $\left[\overline{e_{a, k_{v}}}\right]_{\text {upper }}$ are the upper and lower limits of the favorable range. Equation 17 signifies that if vibrational energy of each reactant lies in their favorable range, the forward reaction will occur, otherwise the reaction will not occur. 
$R_{f a v}=\left[\overline{e_{j, k_{v}}}\right]_{\text {lower }} \leq \overline{\bar{e}_{j, k_{v}}} \leq\left[\overline{e_{j, k_{v}}}\right]_{\text {upper }}$

Based on postulate 2, the kinetics of reaction depends on where the vibrational energy of reactants lies in their favorable ranges of equation 17. Certain vibrational energies corresponds to faster kinetics of reaction, and these frequencies always lies in corresponding favorable ranges of the reactants. Based on postulate 3 , the vibrational frequency of product in this case $\overline{e_{c, k_{V}}}$ of $c$ depends on the vibrational frequencies of reactants as well as the Temperature. If the product undergoes further reaction, that reaction will follows the same postulates. All the oscillation frequencies or vibrational energies are quadratic function of temperature of the surrounding environment of the nucleus. This is a significant insight into biological reactions, as varying the temperature of environment, one can manipulate the reactions and reaction kinetics of the processes in the nucleus. 


\section{Aspects of Evolution Theory at Molecular Level}

The postulates of evolution theory can be qualitatively explain at molecular level using vibrational energy framework and the postulates of section 3. Consider three systems A, B and C containing similar reactant molecules denoted by $\mathrm{a}, \mathrm{b}, \mathrm{c}$ and $\mathrm{d}$, shown in figure 1 . At time $\mathrm{t}=0$, all the reactant molecules of same type in the three systems have same energies given by $e_{I_{j}}$ where $I$ represents the system, $j$ represents the molecule type. At time $\mathrm{t}=0$, the systems are subject to three different temperatures $\mathrm{T} 1, \mathrm{~T} 2$ and $\mathrm{T} 3$. The systems are left to evolve, at these temperature conditions.
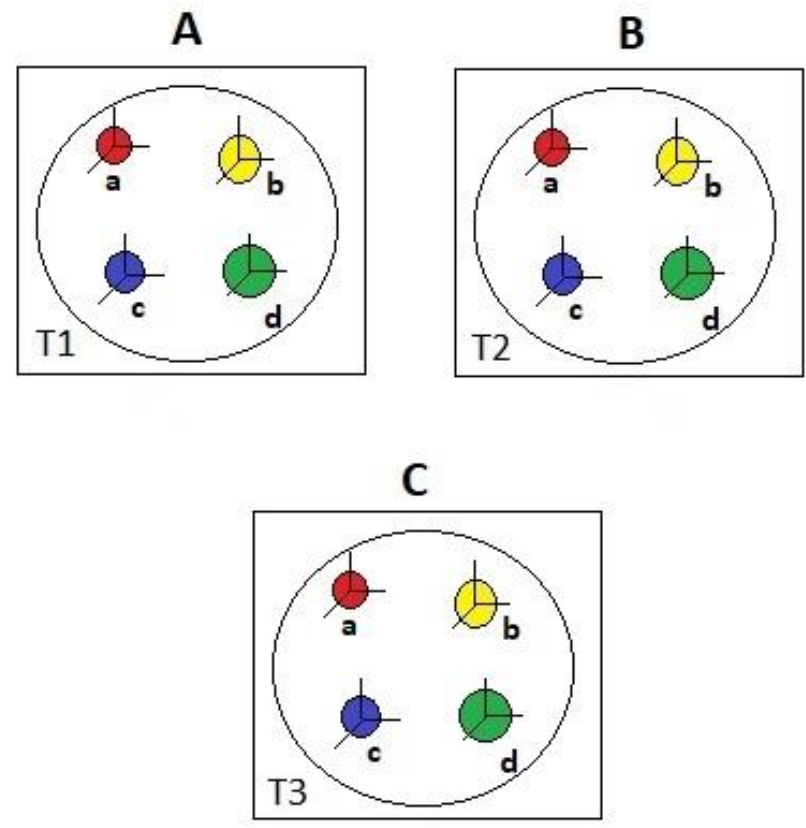

Figure 1: Three systems A, B and C at Time $\mathrm{t}=0$, with Temperatures T1, T2 and T3, respectively.

Based on the temperature values the fluctuations in the vibrational energies of the molecules will be different for different systems given by equation 18 , where $\overline{I_{I_{x}, k_{V}}}$ is the vibrational energy of type $\mathrm{x}$ 
molecule, $T_{i}$ is the system temperature corresponding to the system and $\sum_{I_{j \neq x}}^{n} \overline{e_{I_{j}, k_{V}}}$ is the sum of average vibrational energies of other molecule types. $K_{I}$ is the sum of chemical potential energy, translational and rotational kinetic energies given by equation 4 . The values of chemical potential energy, translational and rotational kinetic energies changes with temperature, but the fluctuations in these energies are kept constant for simplicity.

$\overline{e_{I_{x}, k_{V}}}=k_{B} T_{i} \frac{\partial \ln Z}{\partial T_{i}}-K_{I}-\sum_{I_{j \neq x}}^{n_{j}} \overline{e_{I_{j}, k_{V}}}$

The systems are left to evolve at different temperatures, the vibrational energies of the molecules will be different in different systems. Based on the postulates of section 3, the reactions between molecules only occurs if the vibrational energies of the system lies in the favorable range, and kinetics of the reaction depends on where the molecules lie in the vibrational energy ranges. Based on this premise, the reaction potentials of certain reactions in the systems are favorable and certain reactions are unfavorable. The systems will evolve according to the favorable reactions, so at different environmental conditions, in this case temperature. At a later time $\mathrm{t}$, the systems are evolved differently, with different products depending on the favorable reactions.

Using this premise, the evolution of biological life from simple molecules can be qualitatively explained. The arrangement of simple atoms and molecules constitutes complex molecules and these complex molecules constitutes a biological cell. The arrangement of simpler molecules into complex molecules depends on the favorable reactions according to the vibrational energies of molecules, Furthermore, the vibrational energies of the complex molecules is also dependent on the vibrational energies of simpler molecules as well as the temperature. One such complex molecule is the chromosome in the cell. During mitosis, which in turns depends on the vibrational energies of the molecules involved, the cell divides into following a well-researched cell cycle ${ }^{4}$. These cells then 
constitutes tissues and following the same logic the living species. At molecular level, the effects of temperature on the vibrational energies of molecules cannot be ignored, which in turns change the reaction potential of certain reactions at molecular level.

\subsection{Mutation at Molecular Level}

The process of mutation as a result of molecular mutagen can be explained at molecular level using the premise of this paper. Consider a sequence of simple molecules in a DNA and a mutagen $b^{\prime}$ as shown in Figure 2. The mutagen $b^{\prime}$, reacts with molecule $b$ to alter the DNA structure, as shown in equation 19, where $T$ is the temperature of the surrounding. The reaction will only occur if the vibrational energies of the reacting molecules lies in the favorable energy range. Furthermore, the kinetics of the reaction depends on where the energy lies in their particular favorable energy ranges.

$-[a-b-c-d]-+b^{\prime} \stackrel{T}{\rightarrow}-\left[a-b+b^{\prime}-c-d\right]-$
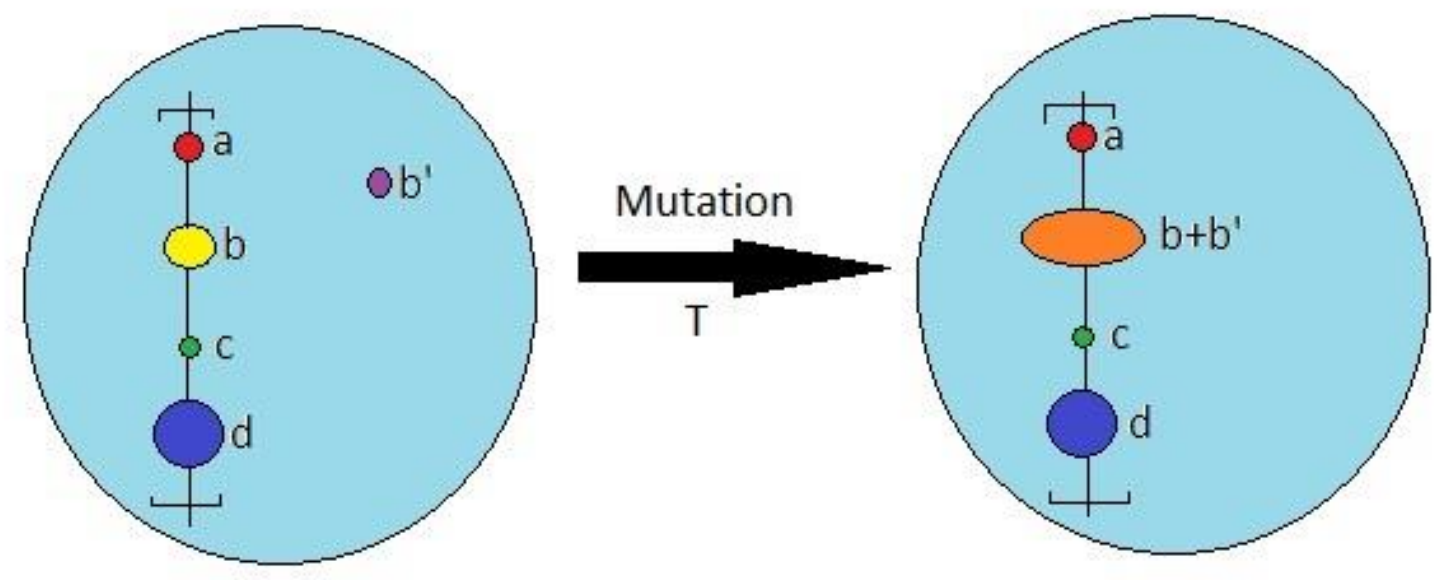

Figure 2. The molecular mutation reaction at an arbitrary sequence of DNA with Surrounding Temperature T.

If reaction potential of mutation reaction is favorable, the molecular structure of DNA will alter, as well as the vibrating frequency of DNA (postulate 3). If the conditions are favorable for the mitosis 
of the nucleus, which has a certain vibrational energy of its own, the resulting DNA replication will be structurally different. The kinetics of the cell cycle is also dependent on the vibrational energy of the nucleus. This qualitatively explains why cell cycle times vary for different cells.

\subsection{Natural Selection}

Darwin's evolution theory postulates that organisms are able to adapt to the environment. At molecular level this postulate can be qualitatively explained by the premise of this paper. Environment in this case, temperature fluctuates the vibrational frequencies of the molecules in the nucleus, making reaction potentials of certain reactions involving these molecules favorable following the postulates of section 3. These favorable reactions results in mutations at molecular level which translates to the adaptability of organisms and species. It is the environment in this case temperature which is responsible to the organisms to adapt. Based on this premise, natural selection is just the favorable reactions caused by the environment at molecular level. If the reactions are not favorable according to the environment (temperature) at molecular level, the adaptability of species diminishes and in the long run species becomes extinct. 


\subsection{Cancerous Mutations}

This section discusses the cancerous mutations of normal cells by carcinogens, at molecular level using the framework of this paper. It has been established that the mutations at molecular level occurs if the vibrational energies of reacting molecules lies in the favorable energy range. Likewise, the mutation by carcinogens follows the same postulates of section 3. Furthermore, the kinetics of mutation depends on the vibrational energies of the molecules. The resulting mutated DNA has its own vibrational energy, which is the function of environment in this case, the Temperature. The replication of cancerous mutated DNA is also effected based on the vibrational frequencies, and the cell cycle time of cancerous cells varies according, which can be faster or slower depending on the favorable conditions. This qualitatively explains why mutations in certain cells occur and not in others and why the growth rates of cancers varies among different organisms and species. Environmental conditions are of paramount importance, as at molecular level they make certain reactions favorable than others. The structure and dynamics of cancer cells is different from normal cells based on the vibrational frequencies of molecules it is made up of. This aspect can be used to understand cancer in general and certainly, treatments and technologies can be developed. Some work is still ongoing in this regard ${ }^{5,6}$. 


\subsection{Astrobiology}

The framework of this article has direct implications on the field of astrobiology. The molecules in astrological environments have different vibrational energies and the reaction potentials of favorable conditions are different, so the arrangement of simple molecules will evolve differently. The current status of biological life in astronomical conditions is bleak, so the authors argue that reaction potentials even with the right ingredient molecules necessary for life on earth might not constitute biological life. And if somehow the favorable reactions by the astronomical environments can constitute life, the evolution of molecules constituting organisms may be drastically different than that on earth. 


\section{Conclusion}

To conclude, it has been shown that cytoplasmic temperatures have profound effect on the vibrational energies of molecules in nucleus, which in turn change the reaction potential of molecules making some reactions favorable. These favorable reactions of simple molecules constitute complex molecules which in turns constitute organisms. The favorable reactions at molecular explains the evolution in organisms, and natural selection at molecular level is mere favorable reactions allowed by the environmental conditions in this study Temperature. This simplest framework has direct implication in understanding mutations causing cancer at molecular framework. The future prospect is to make models more complex including the effects on other forms of energies. 


\section{References}

1. Scott-Phillips, T. C., Laland, K. N., Shuker, D. M., Dickins, T. E., West, S. A., (May 2014). The Niche Construction Perspective: A Critical Appraisal. Evolution. 68 (5): 1231-1243. DOI: https://doi.org/10.1111\%2Fevo.12332

2. Hall, B. K., Hallgrímsson, B., (2008). Strickberger's Evolution (4th ed.). Sudbury, Massachusetts: Jones and Bartlett Publishers. ISBN 978-0-7637-0066-9. LCCN 2007008981. OCLC 85814089.

3. Voet, D., Voet, J. G., Pratt, C. W., (2016). Fundamentals of Biochemistry: Life at the Molecular Level (Fifth ed.). Hoboken, New Jersey: John Wiley \& Sons. ISBN 978-1-11891840-1. LCCN 2016002847. OCLC 939245154.

4. Wang J. D., Levin, P. A., (November 2009). Metabolism, cell growth and the bacterial cell cycle. Nature Reviews. Microbiology. 7 (11): 822-7. doi:10.1038/nrmicro2202.

5. Or, M., Kimmel, E., (June 2009). Modeling linear vibration of cell nucleus in low intensity ultrasound field. Ultrasound Med Biol. 35(6): 1015-25. Doi: https://doi.org/10.1016/j.ultrasmedbio.2008.11.011

6. Fraldi, M., Cugno, A., Deseri, L., Dayal, K,, Pugno, N. M., (2015) A frequency-based hypothesis for mechanically targeting and selectively attacking cancer cells. J. R. Soc. Interface 12: 20150656. Doi: http://dx.doi.org/10.1098/rsif.2015.0656 


\section{Appendix A}

This appendix presents the derivation of the exponential probability distribution of energy state fluctuations of subsystem 1, given by equation 1 . Based on the assumptions of section 2.1 , the probability $P\left(E_{N}\right)$, i.e. the number of microstates of the total system such that the energy of subsystem 1 is $E_{N}$ over the total number of microstates is given by equation A-1

$P\left(E_{N}\right)=\frac{\Omega_{C} E_{C}}{\Omega_{t o t} E_{t o t}}$

Taking natural logarithm of equation A-1, and using $E_{\text {tot }}=E_{N}+E_{C}$ it can be written as equation A2

$\ln P\left(E_{N}\right)=\ln \Omega_{C}\left(E_{t o t}-E_{N}\right)-\ln \Omega_{t o t} E_{t o t}$

Since it has been assumed $E_{\text {tot }} \gg>E_{N}$, the first term of equation A-2 is expanded using Taylor series at energy $E_{t o t}$. The resulting equation is given by equation A-3, where $H . T$ are higher order terms.

$\ln P\left(E_{N}\right)=\ln \Omega_{C} E_{t o t}-E_{N} \frac{\partial \ln \Omega_{C} E_{t o t}}{\partial E_{t o t}}+H . T-\ln \Omega_{t o t} E_{t o t}$

Neglecting higher order terms and representing constant terms with $m$, where $m$ is given by equation A-4, the equation can be written as A-5, where $\beta$ is he thermodynamic-beta defined as $\beta=$ $\frac{\partial \ln \Omega_{C} E_{t o t}}{\partial E_{t o t}}=\frac{1}{k_{B} T}$, from thermodynamic literature.

$m=\ln \Omega_{C} E_{t o t}-\ln \Omega_{t o t} E_{t o t}$

$\ln P\left(E_{N}\right)=m-E_{N} \beta$

Taking natural logarithm of equation A-5, the probability $P\left(E_{N}\right)$ can be written as equation A-6, with $s=e^{m}=\frac{\Omega_{C} E_{t o t}}{\Omega_{t o t} E_{t o t}}$, which is the ratio of microstates of subsystem 2 with energy $E_{\text {tot }}$ and total number of microstates. 
$P\left(E_{N}\right)=s . e^{-E_{N} \beta} \quad(\mathrm{A}-6)$

Assuming, the energy states of nucleus are continuous, the normalization factor is given by the continuous partition function $\mathrm{Z}$ given by equation $\mathrm{A}-7$, where $\rho\left(E_{N}\right)$ is the energy state density. The normalized probability $P\left(E_{N}\right)$ of energy state of subsystem 1 is given by equation A-8.

$$
\begin{aligned}
& Z=\int e^{-E_{N} \beta} \rho\left(E_{N}\right) \partial\left(E_{N}\right) \\
& P\left(E_{N}\right)=\frac{s \cdot e^{-E_{N} \beta}}{\int e^{-E_{N} \beta} \rho\left(E_{N}\right) \partial\left(E_{N}\right)}
\end{aligned}
$$

